\title{
Enhancement of Antibacterial Effect of Quaternary Ammonium with Inorganic Nanosheets against Enterobacter cloacae
}

\author{
Eri Yoshida ${ }^{1}$, Murray Lawn ${ }^{1}$, Takeshi Nagayasu ${ }^{1}$ and Kai Kamada ${ }^{2, *}$ \\ ${ }^{1}$ Graduate School of Biomedical Sciences and ${ }^{2}$ Graduate School of Engineering, \\ Nagasaki University, Nagasaki 852-8521, Japan
}

\footnotetext{
* Corresponding author

Department of Chemistry and Materials Engineering

Graduate School of Engineering, Nagasaki University

1-14, Bunkyo-machi, Nagasaki 852-8521, Japan

Tel/Fax: +81-95-819-2667

Email:kkamada@nagasaki-u.ac.jp
} 


\begin{abstract}
To suppress nosocomial infections, numerous studies of quaternary ammonium cations $\left(\mathrm{R}_{4} \mathrm{~N}^{+}\right)$to improve the antibiotic properties have been investigated. However, most of them reported developments of novel organic or polymeric materials with $\mathrm{R}_{4} \mathrm{~N}^{+}$. To pioneer antibacterial inorganic materials hybridized with $\mathrm{R}_{4} \mathrm{~N}^{+}$, a colloidal solution of metal oxide nanosheets, which have a small particle size (typically less than 10 $\mathrm{nm}$ ), is considered to be a suitable option because oxide nanosheets with a negative surface charge strongly interact $\mathrm{R}_{4} \mathrm{~N}^{+}$. Herein, we demonstrate for the first time that the high antibacterial/bactericidal effects of titanate nanosheets (TNS) adsorbing tetramethylammonium (TMA-TNS) or tetrabultylammonium ions (TBATNS). Their antibacterial effects against Enterobacter cloacae were evaluated using a colony forming unit (CFU) counting method. The results showed that the synthesized TNS composites had superior antibacterial and bactericidal effects to those of free $\mathrm{R}_{4} \mathrm{~N}^{+}$and TBA-TNS exhibited the strongest effect (69\% CFU reduction compared with that of free $\mathrm{TBA}^{+}$and $98 \% \mathrm{CFU}$ reduction compared with the control) among the samples examined. Dark incubation was employed to ensure photocatalytic reaction of semiconducting TNS did not contribute to the process. Compared with $\mathrm{TiO}_{2}$ spherical particles, such high bactericidal effect would be induced by a synergistic function of $\mathrm{TBA}^{+}$and TNS, which physically damages bacteria due to long hydrophobic alkyl chains and an anisotropic nanocrystalline structure with sharp edges, respectively.
\end{abstract}

Keywords: Titanate nanosheets; Quaternary ammonium compounds; Enterobacter cloacae; Antibacterial; Bactericidal

\title{
1 Introduction
}

For several decades, bacterial infection has been identified as the source of many serious problems in the field of medical treatment. Enterobacter is well known to be a representative bacterial species of nosocomial and opportunistic pathogens during the last three decades in hospital wards, and it becomes an issue of bacteremia, endocarditis, septic arthritis and so on (Davin-Regli and Pagès 2015). The pathogenic prevalence has been reported even in recent years and this species is highly ranked as a common microorganism causing bloodstream infection, healthcare-associated intra-abdominal infection and pneumonia 
in ICUs (intensive care units) (Pfaller et al. 2017; Zalacain et al. 2016; Pfaller et al. 2018; Poorabbas et al. 2015). At present, Enterobacter cloacae (E. cloacae) employed in this study is the most frequently observed clinical isolate among Enterobacter species and tends to contaminate various medical, intravenous and other hospital devices (Davin-Regli and Pagès 2015). Furthermore, the infection caused by E. cloacae results in the highest mortality rate among all Enterobacter infections, although the bacteria are not directly related to the primary chronic diseases that the patients are afflicted with (Deal et al. 2007; Ye et al. 2006).

To reduce these nosocomial pathogens, various kinds of antibiotics have been developed. However, it occasionally causes production of multi-drug resistant bacteria due to acquirement of numerous genetic mobile elements (Davin-Regli and Pagès 2015). The antibiotic resistance is also a critical problem against Enterobacter species as with other nosocomial pathogens (Pfaller et al. 2017; Zalacain et al. 2016; Pfaller et al. 2018; Poorabbas et al. 2015). Considering difficulties to create new antibiotics and a requirement to avoid further drug resistance, reduction in a quantity of drug consumption and intensification of medicinal effect of current antibiotics without inducing genetic transition of bacteria are still ongoing important challenges.

The purpose of this study is to develop a new antibacterial composite material against E. cloacae. Herein, we use quaternary ammonium cations $\left(\mathrm{R}_{4} \mathrm{~N}^{+}\right)$as antibiotics. $\mathrm{R}_{4} \mathrm{~N}^{+}$is common antibiotics and disinfectants in nosocomial use and the food industry due to their wide antimicrobial spectra against both of Gram-negative and Gram-positive bacteria as well as against fungi, viruses and algae (Jiao et al. 2017; Souza et al. 2007; Maeda et al. 1999). To improve the antibacterial properties or to pioneer new application fields of these antibiotics, numerous studies have been reported. However, most of them focused on synthesis of new antibacterial organic molecules (Kourai et al. 2006; Chanawanno et al. 2010; Shtyrlin et al. 2016) or polymerization with $\mathrm{R}_{4} \mathrm{~N}^{+}$(Jiao et al. 2017; Farah et al. 2015; Zaltsman et al. 2016). That is, there is few report with respect to composites between metal or inorganic materials and antibiotic molecules.

In this study, we attempted to develop novel antibacterial materials composed of metal oxide nanosheets and $\mathrm{R}_{4} \mathrm{~N}^{+}$. Oxide nanosheets generally have a negative surface charge in an aqueous solution over a wide range of $\mathrm{pH}$, and hence the colloidal solution of nanosheets exhibits high dispersion stability in the presence of $\mathrm{R}_{4} \mathrm{~N}^{+}$such as tetrabutylammonium ions (Ohya et al. 2002). This implies the colloidal solution of oxide nanosheets including $\mathrm{R}_{4} \mathrm{~N}^{+}$could be useful as a disinfectant. Titanium has been chosen from a variety of 
possible metals to produce oxide nanosheets, as it would be suitable for medical use because of its biocompatibility. This study demonstrates the superior antibacterial and bactericidal effects of titanate nanosheets (TNS) adsorbing $\mathrm{R}_{4} \mathrm{~N}^{+}$as compared to free $\mathrm{R}_{4} \mathrm{~N}^{+}$(not adsorbed to TNS) and discusses a potential mechanism by comparison with spherical titanium oxide particles.

\section{Materials and methods}

\subsection{Materials}

Aqueous solutions of tetramethylammonium hydroxide (TMAOH, $25 \mathrm{wt} \%)$, tetrabutylammonium hydroxide (TBAOH, $40 \mathrm{wt} \%$ ) and liquid titanium(IV) tetraisopropoxide (TTIP, 99.99\%) were purchased from Sigma-Aldrich. An aqueous solution of hydrochloric acid (37\%) and titanium(IV) oxide $\left(\mathrm{TiO}_{2}\right)$ powder were purchased from Kishida Chemical. All chemicals were used without further purification. Ultrapure water (specific resistivity: $>18.2 \mathrm{M} \Omega \mathrm{cm}$ ) produced by a Millipore Milli-Q purification system was used throughout this study.

\subsection{Synthesis of TNS}

Colloidal solutions of TNS adsorbing $\mathrm{R}_{4} \mathrm{~N}^{+}$were synthesized through a hydrolysis of TTIP (Ohya et al. 2002). Concretely, $0.603 \mathrm{ml}$ of the TMAOH aq. was mixed with ultrapure water $(3.897 \mathrm{ml})$. Then $0.500 \mathrm{ml}$ of TTIP was added to the solution to hydrolyze TTIP followed by ultrasonication for 20 min, where the molar ratio of Ti for $\mathrm{TMA}^{+}$was set to be unity. The obtained suspension including white precipitates was aged at $60^{\circ} \mathrm{C}$ for $2 \mathrm{~h}$ with gentle shaking, then a colorless and transparent colloidal solution was produced. The resulting colloidal solution was dialyzed against pure water using a membrane filter (molecular weight cut off (MWCO): $3 \mathrm{kDa}$ ) to remove by-products such as isopropanol. The dialysis was repeated several times until the $\mathrm{pH}$ reached 7.5. The resulting colloidal solution is referred to below as TMA-TNS. In addition, a colloidal solution of TNS including TBA (TBA-TNS) was also prepared with TBAOH aq. (1.095 ml), TTIP $(0.500 \mathrm{ml})$ and $\mathrm{H}_{2} \mathrm{O}(3.405 \mathrm{ml})$ in the same manner as TMA-TNS.

\subsection{Material characterization}


Particle sizes of TNS composites in the colloidal solutions were evaluated by dynamic light scattering (DLS, Malvern, HPPS501). The particle size of $\mathrm{TiO}_{2}$ powder was obtained using scanning electron microscopy (SEM, JEOL, JCM-5700ME). X-ray diffraction analysis (XRD, Rigaku, RINT2000VL) was utilized to determine a crystal structure of the TNS composites. The measurement was conducted for thin film samples after drying the colloidal solutions on glass plates. Raman spectroscopy (JASCO, NRS3000) was adapted to evaluate a crystal structure of TNS and concentration of $\mathrm{R}_{4} \mathrm{~N}^{+}$in the colloidal solutions. Concentration of titanium in the colloidal solutions was determined using colorimetry. A concentrated $\mathrm{H}_{2} \mathrm{SO}_{4}$ solution was added to the colloidal solution and then heated at $80^{\circ} \mathrm{C}$ for $1 \mathrm{~h}$ to dissolve the solid TNS. After cooling down to room temperature, the solution was colored by adding an $\mathrm{H}_{2} \mathrm{O}_{2}$ solution to produce a peroxocomplex of Ti. As the peroxocomplex has a maximum absorbance at $\lambda=409 \mathrm{~nm}$ (Inada et al. 2006), the concentration of Ti was determined by measuring the absorbance with a microplate reader (BioTek, Synergy HT) using a calibration curve that had been created using several Ti standard solutions with known concentrations.

\subsection{Antibacterial activity}

A strain of Enterobacter cloacae (E. cloacae, ATCC 13047, Microbiologics), a microorganism typically responsible for nosocomial infections, was used to investigate the antibacterial effect of materials. All apparatus and reagents utilized for bacterial tests were sterilized before use. Sterilization was performed at $121^{\circ} \mathrm{C}$ for $20 \mathrm{~min}$ by using an autoclave apparatus (SK medical, LABOCLAVE).

Prior to each experiment, the bacterial cultures were refreshed on LB agar medium (Sigma-Aldrich), then cultured overnight in an incubator at $37^{\circ} \mathrm{C}$. Solutions to investigate antibacterial performance were prepared by mixing (a) $1800 \mu 1$ of LB broth liquid microbial growth medium (Sigma-Aldrich), (b) $500 \mu 1$ of sample solution (TMA-TNS, TBA-TNS) and (c) $200 \mu \mathrm{l}$ of a bacterial suspension in sterilized ultrapure water $(1 \mathrm{mg} / \mathrm{mL})$. A suspension of commercial $\mathrm{TiO}_{2}$ powder or aqueous solutions of free $\mathrm{TMA}^{+}$or $\mathrm{TBA}^{+}$ions, which were prepared by neutralization of TMAOH or $\mathrm{TBAOH}$ with a concentrated hydrochloric acid solution, were also used instead of (b)-solution to clarify the influence of TNS on bacterial growth (referred below as $\mathrm{TiO}_{2}$, 
TMA-Cl and TBA-Cl, respectively). Ultrapure water was used instead of (b)-solution, referred below as "the control".

Bacterial growth in the mixed solutions was monitored by measuring optical density (OD) of visible light (turbidimetric method), because the OD is related to the number of microorganisms. During incubation of the mixed solution at $37^{\circ} \mathrm{C}$, changes in $\mathrm{OD}$ were monitored with visible light at $\lambda=600 \mathrm{~nm}$ using a microplate reader.

A colony forming units ( $\mathrm{CFU}$ ) counting method was also used to evaluate antibacterial effect of materials. Sample solutions were produced in the identical manner to the turbidimetric method. The test solutions were incubated at $37^{\circ} \mathrm{C}$ for certain periods $\left(5,10\right.$ and $22 \mathrm{~h}$ ), then diluted to $10^{1} \sim 10^{13}$ times with sterilized water. $10 \mu \mathrm{l}$ of each diluted solution was spread on LB agar $\left(13 \mathrm{~cm}^{2}\right)$ in a petri dish. The petri dishes were incubated at $37^{\circ} \mathrm{C}$ for $24 \mathrm{~h}$ to produce visible colonies. The number of colonies on the dishes were visually counted, and then CFUs in the original solutions were calculated using the dilution factors. All incubation processes in this work were performed in darkness to avoid photocatalytic antibacterial effect of titanium oxide.

\subsection{Statistical analysis}

Experiments conducted in this study were repeated at least in triplicate $(n=3)$. Results were reported as mean values and each standard deviation is shown by an error bar. The data of CFU counting tests was analyzed by one-way analysis of variance (ANOVA) and Dunnett's post hoc test. The level of significance was set as $5 \%$.

\section{Results}

\subsection{Material characterization}

Several physical properties of synthesized TNS colloidal solutions are shown in Fig. 1. Fig. 1a is a photograph of the TBA-TNS colloidal solution. Although the solution is colorless and transparent, a red laser beam directed is clearly scattered due to presence of tiny TNS. The particle sizes of the TNS composites were measured using the dynamic light scattering method (DLS). Fig. 1b shows the particle size distribution curves 
of TMA-TNS and TBA-TNS. The curves consist of a single broad peak with a small shoulder, indicating monodispersion of the TNS composites and no additional peaks being observed exceeding $10 \mathrm{~nm}$. The mean particle size was calculated to be 2 and $4 \mathrm{~nm}$ for TMA-TNS and TBA-TNS, respectively.

Fig. 1c shows the X-ray diffraction (XRD) patterns of TMA-TNS and TBA-TNS thin films after drying the colloidal solutions on glass plates. During the drying, TNS accommodates $\mathrm{R}_{4} \mathrm{~N}^{+}$in the interlayer space and hence constructs a periodical layered structure. On one hand, Raman spectra of TMA-TNS and TBA-TNS colloidal solutions (Fig. 2a) validated formation of TNS with an akin crystal structure to tetratitanate $\left(\mathrm{Ti}_{4} \mathrm{O}_{9}{ }^{2-}\right)$ (Ohya et al. 2002). Due to the large two-dimensional (in-plane) size of TNS for the thickness $(0.75 \mathrm{~nm})$, both XRD patterns have several diffraction lines assigned to the (n00) planes of tetratitanate that are perpendicular to the two-dimensional layers. The $d$-spacings of the (200) planes $\left(d_{(200)}\right)$ calculated using a Bragg equation, which correspond to the sum of interlayer distance and thickness of a single tetratitanate nanosheet, were $1.3 \mathrm{~nm}$ for TMA-TNS and $1.7 \mathrm{~nm}$ for TBA-TNS.

The Raman spectra were employed to determine the concentration of $\mathrm{R}_{4} \mathrm{~N}^{+}$in the colloidal solutions. Standard TMA-Cl and TBA-Cl solutions were also measured in order to prepare calibration curves. Characteristic Raman bands of TMA $\left(721-772 \mathrm{~cm}^{-1}\right)$ and $\mathrm{TBA}^{+}\left(850-964 \mathrm{~cm}^{-1}\right)$ were utilized for quantitative analysis. As shown in Fig. 2b, the band peaks of standard solutions linearly increase with an increase in the concentration of $\mathrm{R}_{4} \mathrm{~N}^{+}$within the ranges examined. As a result, the concentrations of $\mathrm{TMA}^{+}$in the TMA-TNS and $\mathrm{TBA}^{+}$in the TBA-TNS were estimated to be 0.26 and $0.24 \mathrm{M}$, respectively. In addition, concentrations of $\mathrm{Ti}^{4+}$ in the colloidal solutions evaluated by the colorimetric method were estimated to be 1.14 $\mathrm{M}$ and $0.73 \mathrm{M}$ for TMA-TNS and TBA-TNS, respectively.

\subsection{Turbidimetric assay}

In this work, quaternary ammonium ions $\left(\mathrm{R}_{4} \mathrm{~N}^{+}\right)$adsorbed on TNS are used as antibiotics. Prior to investigation of antibacterial activity of TMA-TNS and TBA-TNS, influence of free $\mathrm{R}_{4} \mathrm{~N}^{+}$(not adsorbed on TNS) on bacterial growth was studied using the turbidimetric method. Fig. 3a shows changes in optical density $(\mathrm{OD}, \lambda=600 \mathrm{~nm})$ of bacterial suspensions with several concentrations of $\mathrm{TMA}^{+}(0.10 \mathrm{M})$ and $\mathrm{TBA}^{+}(0.01$, 0.05 and $0.10 \mathrm{M}$ ) together with a control sample. The OD is related to the number of bacterial cells in the 
suspensions, and hence it is noted that the monitoring of OD indirectly follows the bacterial growth process. A remarkable difference in $\mathrm{OD}$ between $\mathrm{TMA}^{+}$and $\mathrm{TBA}^{+}$was observed at a fixed concentration $(0.10 \mathrm{M})$. The impact of $\mathrm{TMA}^{+}$on bacterial proliferation was little and the increasing rate of OD was nearly equal to that of the control. In contrast, $\mathrm{TBA}^{+}$suppressed the bacterial growth as OD did not increase at all even after $9 \mathrm{~h}$.

Concentration dependence of $\mathrm{TBA}^{+}$on the proliferation was also examined as plotted in Fig. 3a. The antibacterial effect of $\mathrm{TBA}^{+}$was proportional to the concentration of $\mathrm{TBA}^{+}(\mathrm{OD}$ at $9 \mathrm{~h}$ : control $\sim 0.01 \mathrm{M}>$ $0.05 \mathrm{M}>0.10 \mathrm{M}$ ). Based on additional turbidimetric experiments for other $\mathrm{R}_{4} \mathrm{~N}^{+}$at $0.05 \mathrm{M}$ (Fig. 3b), a positive relationship between alkyl chain length and the antibacterial activity of $\mathrm{R}_{4} \mathrm{~N}^{+}$was confirmed. Especially, no growth was observed in the presence of tetrahexylammonium ions with the longest chains. The solution containing tetrahexylammonium ions was still clear even after $77 \mathrm{~h}$ incubation (Fig. 3c).

\subsection{Antibacterial efficiency of TNS}

By using the CFU counting method, the antibacterial effect of TMA-TNS, TBA-TNS, TMA-Cl and TBA-Cl was examined under the same concentration of $\mathrm{R}_{4} \mathrm{~N}^{+}(0.05 \mathrm{M})$. As shown in Fig. 4a, colonies composed of bacteria formed on the agar and they were counted to estimate CFU in the original suspensions. Firstly, to study influence of TNS on the antibacterial effect, CFUs of TMA-TNS and TBA-TNS was compared with those of TMA-Cl and TBA-Cl. The results after incubation for $5 \mathrm{~h}$ are shown in Fig. $4 \mathrm{~b}$. The initial bacterial number before incubation was $1.0 \times 10^{8} \mathrm{CFU} / \mathrm{ml}$. The CFU of TMA-Cl increased with increasing incubation time as comparable to that of control. The CFU of TMA-TNS seems to maintain the initial bacterial number. Meanwhile, the bacterial growth was markedly suppressed in the presence of $\mathrm{TBA}^{+}$ (TBA-Cl and TBA-TNS). Furthermore, the CFU of TBA-TNS reduced (about 30\%) as compared to that of TBA-Cl ( $p<0.0001$ for both $\mathrm{TBA}^{+}$samples in relation to the control). That is, hybridization with TNS (TMATNS, TBA-TNS) is effective in enhancement of antibacterial effect of $\mathrm{R}_{4} \mathrm{~N}^{+}$. In addition, difference in CFU between the control and each material increased with increasing incubation time for 10 and $22 \mathrm{~h}$.

To clarify the contribution of TNS to the remarkable reduction in the bacterial number as depicted in Fig. $4 \mathrm{~b}$, antibacterial activity of titanium (IV) oxide $\left(\mathrm{TiO}_{2}\right)$ powder was also evaluated through the $\mathrm{CFU}$ counting method. Fig. 4c shows CFUs of E. cloacae suspensions including $\mathrm{TiO}_{2}$ alone or $\mathrm{TiO}_{2}$ mixed with 
TBA-Cl (referred as $\mathrm{TiO}_{2} / \mathrm{TBA}-\mathrm{Cl}$ ) after incubation at $37^{\circ} \mathrm{C}$ for $5 \mathrm{~h}$, where the concentrations of $\mathrm{Ti}^{4+}$ and $\mathrm{TBA}^{+}$were equal to those of TBA-TNS used in Fig. $4 \mathrm{~b}$. The CFUs of $\mathrm{TiO}_{2}$ and control were similar $(p=$ 0.187). Additionally, $\mathrm{TiO}_{2} / \mathrm{TBA}-\mathrm{Cl}$ did not show so much significant reduction in CFU different from TBATNS in Fig. $4 \mathrm{~b}$ ( $p=0.001$ in relation to the control).

\section{Discussion}

The main purpose of this study is to propose a new antibacterial material especially against $E$. cloacae pathogen, which can be used in the medical field.

The single-step hydrolysis reaction of TTIP with an aqueous solution of tetraalkylammonium hydroxide (TMAOH or TBAOH) resulted in formation of a colorless and transparent solution. The colloidal solutions were stable and could be stored for more than 10 months without precipitation. In the colloidal solution, most of TNS would exist as a single tetratitanate layer (Kamada and Soh 2014). Due to a Brownian motion of tiny TNS in the solution, the DLS data obtained (Fig. 1b) could reflect the two-dimensional size of TNS adsorbing $\mathrm{R}_{4} \mathrm{~N}^{+}$as counter ions (Fig. 1d). Furthermore, the XRD patterns clearly indicate that dried TNS thin films have a layered structure because the patterns have peaks assigned only to (n00) planes of tetratitanate (Fig. 1c). The calculated $d$-spacing values of a (200) plane vary according to counter ions $(1.3 \mathrm{~nm}$ for TMA-TNS and $1.7 \mathrm{~nm}$ for TBA-TNS). Considering that both TNS has identical thickness of a tetratitanate layer, it is appeared that the discrepancy between the $d$-spacings is based on variation in interlayer distances. Namely, the intercalation of smaller $\mathrm{TMA}^{+}$resulted in a narrow interlayer distance as compared to larger $\mathrm{TBA}^{+}$. Moreover, the broad diffraction line observed for TMA-TNS suggests the small lateral size of TNS as estimated by DLS.

The concentration of $\mathrm{R}_{4} \mathrm{~N}^{+}$and $\mathrm{Ti}^{4+}$ in each TNS colloidal solution was estimated by Raman spectroscopy and a colorimetric method, respectively. As TNS has a crystal structure similar to tetratitanate $\left(\mathrm{Ti}_{4} \mathrm{O}_{9}{ }^{2-}\right.$ ) (Kamada and Soh 2014), $0.5 \mathrm{~mol}$ of $\mathrm{R}_{4} \mathrm{~N}^{+}$for $1 \mathrm{~mol}$ of $\mathrm{Ti}^{4+}$ are required to maintain electrical neutrality. That is, the molar ratio of $\left[\mathrm{TMA}^{+}\right.$or $\left.\mathrm{TBA}^{+}\right] /\left[\mathrm{Ti}^{4+}\right]$ should be 0.5 . However, the actual ratios $(0.23$ for TMA-TNS, 0.33 for TBA-TNS) were lower than estimated values. The residual negative charges of TNS may be compensated with a partial protonation of tetratitanate. 
The antibacterial effects of antibiotics themselves $\left(\mathrm{R}_{4} \mathrm{~N}^{+}\right.$not bounded to TNS) were firstly investigated using the turbidimetric method. As shown in Fig. 3, the higher concentration or the longer alkyl chain length of $\mathrm{R}_{4} \mathrm{~N}^{+}$brought an increased antibacterial effect. According to the previously reported mechanism, long hydrophobic alkyl chains of $\mathrm{R}_{4} \mathrm{~N}^{+}$can penetrate into peptidoglycan cell wall and inner membrane, imposing deformational stress on the bacterial cells (Kim et al. 1997). In addition, other research papers also mention the effectiveness of hydrophobic chains in permeability of cell membrane (Chen et al. 2014; Zhang et al. 2018).

To confirm the antibacterial activity of TNS, the CFU counting method was used (Fig. 4). The turbidimetric method counts both dead and living bacterial cells, while the CFU counting method can evaluate the latter only. Noting that the CFU of the TMA-Cl sample was almost equivalent to that of the control (Fig. 4b), implies that $\mathrm{TMA}^{+}$did not behave as a potent inhibitor and the result is not conflicted with the data shown in Fig. 3a. Moreover, as expected, the samples with $\mathrm{TBA}^{+}$(TBA-Cl and TBA-TNS) exhibit superior antimicrobial activity to $\mathrm{TMA}^{+}$(TMA-Cl and TMA-TNS). In addition, since the CFUs of $\mathrm{TBA}^{+}$samples reduced from the initial bacterial number, $\mathrm{TBA}^{+}$samples must be bactericidal. Furthermore, TMA-TNS and TBA-TNS exhibited more prominent bacterial growth suppression than free $\mathrm{R}_{4} \mathrm{~N}^{+}$(TMA-Cl and TBA-Cl), hence it is expected that TNS possesses antibacterial property even without antibiotics such as $\mathrm{R}_{4} \mathrm{~N}^{+}$.

To reveal the positive contribution of TNS, antibacterial activity was also examined for a mixture of spherical $\mathrm{TiO}_{2}$ powder and $\mathrm{TBA}-\mathrm{Cl}\left(\mathrm{TiO}_{2} / \mathrm{TBA}-\mathrm{Cl}\right)$. According to $\mathrm{SEM}$ observation, the mean particle size of $\mathrm{TiO}_{2}$ is about $200 \mathrm{~nm}$. Some literatures have already reported photocatalytic antibacterial effects of $\mathrm{TiO}_{2}$ under UV light illumination (Kikuchi et al. 1997; Kubacka et al. 2014). As a matter of course, $\mathrm{TiO}_{2}$ did not exhibit any significant antibacterial effect during the dark incubation in the present study (Fig. 4c). When comparing the results of TBA-TNS ( $98 \%$ CFU reduction to the control, Fig. 4b) and $\mathrm{TiO}_{2} / \mathrm{TBA}-\mathrm{Cl}(69 \% \mathrm{CFU}$ reduction to the control, Fig. 4c), it was confirmed that TBA-TNS exhibited prominent antibacterial activity. The excellent antibacterial efficiency of TBA-TNS may be attributed to a synergistic function of TNS and TBA ${ }^{+}$. Recently, Ivanova et al. have reported that sharp nanopillars of silicon physically damage microorganisms (Ivanova et al. 2013). Therefore, highly anisotropic and rigid TNS would also exhibit bactericidal effectiveness through the similar manner. 
The antibacterial and/or bactericidal mechanisms of TBA-TNS and $\mathrm{TiO}_{2} / \mathrm{TBA}-\mathrm{Cl}$ are illustrated in Fig. 5. The negatively charged outer membrane of bacteria attracts $\mathrm{TBA}^{+}$adsorbed on TNS, then $\mathrm{TBA}^{+}$with long hydrophobic alkyl chains damages cell walls and inner membrane (Fig. 5a), while TMA $^{+}$with short alkyl chains cannot harm E. cloacae. Due to the morphological anisotropy, thin and rigid TNS may also induce structural deformation of cells (Fig. 5b). This prediction is based on the results that TMA-TNS and TBA-TNS had greater antibacterial effects compared with TMA-Cl and TBA-Cl, respectively (Fig. 4b). Thus, hybridization with TNS and $\mathrm{TBA}^{+}$affected bacteria as an enhanced antimicrobial material owing to each useful property together, similarly to a conjugated multifunctional nano-material (Chen et al. 2012). On the other hand, in the case of $\mathrm{TiO}_{2} / \mathrm{TBA}-\mathrm{Cl}, \mathrm{TBA}^{+}$would damage bacteria in the same manner as TBA-TNS (Fig. 5c). However, spherical $\mathrm{TiO}_{2}$ particles did not cause significant damage to bacterial cell wall (Fig. 5d). Moreover, when TBA-Cl and $\mathrm{TiO}_{2} / \mathrm{TBA}-\mathrm{Cl}$ samples were compared, the latter exhibited lower antibacterial effect (Fig. 4). This result indicates that relatively large $\mathrm{TiO}_{2}$ particles interfere with the interaction between $\mathrm{TBA}^{+}$and bacteria (Fig. 5e). Thus, it is supposed that the improved antibacterial effect by coexistence of TNS is based on their nano-sized anisotropic morphology with sharp edges, a high specific surface area and high dispersibility.

This study focused on development of a novel antibacterial material for application to medical fields. In this work, we proposed TNS as a host material for antibiotics $\left(\mathrm{R}_{4} \mathrm{~N}^{+}\right)$and their composites exhibited superior antibacterial effects compared to antibiotics alone. The transparent colloidal solution of tiny TNS with antibiotics will be practical as a disinfectant, and it possesses a potential to be used as a fine coating source for various kinds of medical devices to suppress bacterial proliferation, because the sheet-like shape of TNS facilitates a periodical laminated structure intercalated with $\mathrm{R}_{4} \mathrm{~N}^{+}$(Fig. 1d). In fact, other researchers have reported dense coating of nanosheets using a Langmuir-Blodgett technique or a layer-by-layer technique (Muramatsu et al. 2005; Tanaka et al. 2004). Here, we confirmed the antibacterial effect of TNS with antibiotics against E. cloacae, which often causes nosocomial infections and expresses antibiotic resistance. TBA-TNS, which exhibits most excellent performance among the samples investigated in the present study, will also affect Gram-positive bacteria because $\mathrm{TBA}^{+}$has an antibacterial spectrum which includes both Gram- 
positive and Gram-negative bacteria. Furthermore, metal oxide nanosheets will be useful to adsorb not only $\mathrm{R}_{4} \mathrm{~N}^{+}$but also other antibiotics for further improvement of their antibacterial effects.

\section{Conclusion}

This paper studied the antibacterial and/or bactericidal effect of titanate nanosheets (TNS) adsorbing quaternary ammonium cations $\left(\mathrm{R}_{4} \mathrm{~N}^{+}\right)$dispersed in an aqueous solution, where Enterobacter cloacae $(E$. cloacae) was utilized as a representative bacteria that frequently induces nosocomial infections. As a result, TNS with tetrabutylammonium ions (TBA-TNS) was most effective/active among the samples examined and the activity was related to the alkyl chain length of $\mathrm{R}_{4} \mathrm{~N}^{+}$. Although free $\mathrm{TBA}^{+}$ions not adsorbed on TNS (TBA-Cl) also had certain antibacterial effect, the activity was largely enhanced when hybridized with TNS. On the other hand, the mixture of $\mathrm{TBA}^{+}$ions and spherical $\mathrm{TiO}_{2}$ particles did not exhibit significant reduction of bacteria compared to TBA-TNS and TBA-Cl. Taking into consideration that all incubations of bacteria were performed in darkness, photocatalytic reaction of TNS was not a factor. The prominent antibacterial effect of TBA-TNS suggested that anisotropic TNS crystals could physically damage E. cloacae, resulting in bactericidal efficacy. These findings indicate the possibility of TNS as a superior disinfectant by hybridizing with antibiotics. Furthermore, TBA-TNS was highly dispersive in an aqueous solution with high optical transparency and $\mathrm{TBA}^{+}$remained between the interlayers of TNS even after drying, suggesting a potential of TBA-TNS as a transparent fine coating source for healthcare facilities and medical devices to inhibit proliferation of pathogens inducing such as critical hospital infections and surgical site infections.

\section{Acknowledgements}

The authors would like to thank Yuki Nakatsu (Faculty of Engineering, Nagasaki University) for his technical assistance. The present work was partly supported by Research Fellow of Japan Society for the Promotion of Science (JSPS), KAKENHI Grant No. 26410244 and 16J10617.

\section{Conflict of Interest}

The authors declare that they have no conflict of interest. 


\section{References}

Chanawanno K, Chantrapromma S, Anantapong T, Kanjana-Opas A, Fun H (2010) Synthesis, structure and in vitro antibacterial activities of new hybrid disinfectants quaternary ammonium compounds: Pyridinium and quinolinium stilbene benzenesulfonates. Eur J Med Chem. https://doi.org/10.1016/j.ejmech.2010.06.014

Chen M, Hu M, Wang D, Wang G, Zhu X, Yan D, Sun J (2012) Multifunctional Hyperbranched Glycoconjugated Polymers Based on Natural Aminoglycosides. Bioconjugate Chem. https://doi.org/10.1021/bc300016b

Chen M, Zhu X, Yan D (2014) A Controlled Release System for Simultaneous Promotion of Gene Transfection and Antitumor Effects. RSC Adv. https://doi.org/10.1039/c4ra10447a

Davin-Regli A, Pagès J (2015) Enterobacter aerogenes and Enterobacter cloacae; versatile bacterial pathogens confronting antibiotic treatment. Frontiers in Microbiology. https://doi.org/10.3389/fmicb.2015.00392

Deal EN, Micek ST, Ritchie DJ, Reichley RM, Dunne WM, Kollef MH (2007) Predictors of In-Hospital Mortality for Bloodstream Infections Caused by Enterobacter Species or Citrobacter freundii. Pharmacotherapy. https://doi.org/10.1592/phco.27.2.191

Farah S, Aviv O, Laout N, Ratner S, Beyth N, Domb AJ (2015) Quaternary ammonium poly(diethylaminoethyl methacrylate) possessing antimicrobial activity. Colloids Surf, B. https://doi.org/10.1016/j.colsurfb.2015.01.051

Inada M, Kamada K, Enomoto N, Hojo J (2006) Microwave Effect for Synthesis of $\mathrm{TiO}_{2}$ Particles by SelfHydrolysis of $\mathrm{TiOCl}_{2}$. J Ceram Soc Jpn. https://doi.org/10.2109/jcersj.114.814 
Ivanova EP, Hasan J, Webb HK, Gervinskas G, Juodkazis S, Truong VK, Wu AHF, Lamb RN, Baulin VA, Watson GS, Watson JA, Mainwaring DE, Crawford RJ (2013) Bactericidal Activity of Black Silicon. Nat Commun. https://doi.org/10.1038/ncomms3838

Jiao Y, Niu L, Ma S, Li J, Tay FR, Chen J (2017) Quaternary ammonium-based biomedical materials: Stateof-the-art, toxicological aspects and antimicrobial resistance. Prog Polym Sci. https://doi.org/10.1016/j.progpolymsci.2017.03.001

Kamada K, Soh N (2014) Temperature-Controlled Reversible Exfoliation-Stacking of Titanate Nanosheets in an Aqueous Solution Containing Tetraalkylammonium Ions. RSC Adv. https://doi.org/10.1039/C3RA47233G

Kikuchi Y, Sunada K, Iyoda T, Hashimoto K, Akira Fujishima A (1997) Photocatalytic Bactericidal Effect of $\mathrm{TiO}_{2}$ Thin Films: Dynamic View of the Active Oxygen Species Responsible for the Effect. J Photochem Photobiol, A. https://doi.org/10.1016/s1010-6030(97)00038-5

Kim CH, Choi JW, Chun HJ, Choi KS (1997) Synthesis of Chitosan Derivatives with Quaternary Ammonium Salt and their Antibacterial Activity. Polym Bull. https://doi.org/10.1007/s002890050064

Kourai H, Yabuhara T, Shirai A, Maeda T, Nagamune H (2006) Syntheses and antimicrobial activities of a series of new bis-quaternary ammonium compounds. Eur $\mathrm{J}$ Med Chem. https://doi.org/10.1016/j.ejmech.2005.10.021

Kubacka A, Diez MS, Rojo D, Bargiela R, Ciordia S, Zapico I, Albar JP, Barbas C, Martins Dos Santos VAP, Fernández-García M, Ferrer M (2014) Understanding the Antimicrobial Mechanism of TiO2-based Nanocomposite Films in a Pathogenic Bacterium. Sci Rep. https://doi.org/10.1038/srep04134 
Maeda T, Manabe Y, Yamamoto M, Yoshida M, Okazaki K, Nagamune H, Kourai H (1999) Synthesis and Antimicrobial Characteristics of Novel Biocides, 4,4-(1,6-Hexamethylenedioxydicarbonyl)bis(1alkylpyridinium iodide)s. Chem Pharm Bull. https://doi.org/10.1248/cpb.47.1020

Muramatsu M, Akatsuka K, Ebina Y, Wang K, Sasaki T, Ishida T, Miyake K, Haga M (2005) Fabrication of Densely Packed Titania Nanosheet Films on Solid Surface by Use of Langmuir-Blodgett Deposition Method without Amphiphilic Additives. Langmuir. https://doi.org/10.1021/1a050293f

Ohya T, Nakayama A, Ban T, Ohya Y, Takahashi Y (2002) Synthesis and Characterization of Halogen-free, Transparent, Aqueous Colloidal Titanate Solutions from Titanium Alkoxide. Chem Mater. https://doi.org/10.1021/cm0200588

Pfaller MA, Bassetti M, Duncan LR, Castanheira M (2017) Ceftolozane/tazobactam activity against drugresistant Enterobacteriaceae and Pseudomonas aeruginosa causing urinary tract and intraabdominal infections in Europe: report from an antimicrobial surveillance programme (2012-15). J Antimicrob Chemother. https://doi.org/1386-395. doi:10.1093/jac/dkx009

Pfaller MA, Shortridge D, Sader HS, Castanheira M, Flamm RK (2018) Ceftolozane/tazobactam activity against drug-resistant Enterobacteriaceae and Pseudomonas aeruginosa causing healthcare-associated infections in the Asia-Pacific region (minus China, Australia and New Zealand): report from an Antimicrobial Surveillance Programme (2013-2015). Int J Antimicrob. https://doi.org/doi:10.1016/j.ijantimicag.2017.09.016

Poorabbas B, Mardaneh J, Rezaei Z, Kalani M, Pouladfar G, Alami MH, Soltani J, Shamsi-Zadeh A, AbdoliOskooi S, Saffar MJ, Alborzi A (2015) Nosocomial infections: multicenter surveillance of antimicrobial resistance profile of Staphylococcus aureus and Gram negative rods isolated from blood and other sterile body fluids in Iran. Iran J Microbiol 7(3):127-35 
Shtyrlin NV, Sapozhnikov SV, Galiullina AS, Kayumov AR, Bondar OV, Mirchink EP, Isakova EB, Firsov AA, Balakin KV, Shtyrlin YG (2016) Synthesis and Antibacterial Activity of Quaternary Ammonium 4Deoxypyridoxine Derivatives. BioMed Res Int. https://doi.org/10.1155/2016/3864193

Souza AOD, Galetti FCS, Silva CL, Bicalho B, Parma MM, Fonseca SF, Marsaioli AJ, Trindade ACLB, Gil RPF, Bezerra FS, Andrade-Neto M, Oliveira MCF (2007) Antimycobacterial and cytotoxicity activity of synthetic and natural compounds. Quim Nova. https://doi.org/10.1590/s0100-40422007000700012

Tanaka T, Fukuda K, Ebina Y, Takada K, Sasaki T (2004) Highly Organized Self-Assembled Monolayer and Multilayer Films of Titania Nanosheets. Adv Mater. https://doi.org/10.1002/adma.200306470

Ye Y, Li JB, Ye DQ, Jiang ZJ (2006) Enterobacter Bacteremia: Clinical Features, Risk Factors for Multiresistance and Mortality in a Chinese University Hospital. Infection. https://doi.org/10.1007/s15010-006$5038-3$

Zalacain M, Biedenbach DJ, Badal RE, Young K, Motyl M, Sahm DF (2016) Pathogen Prevalence and Antimicrobial Susceptibility Among Enterobacteriaceae Causing Hospital-associated Intra-abdominal Infections in Adults in the United States (2012-2013). Clin Ther. https://doi.org/doi:10.1016/j.clinthera.2016.04.035

Zaltsman N, Kesler-Shvero D, Weiss EI, Beyth N (2016) Synthesis variants of quaternary ammonium polyethyleneimine nanoparticles and their antibacterial efficacy in dental materials. J Appl Biomater Funct Mater. https://doi.org/10.5301/jabfm.5000269

Zhang Y, Zhou Z, Chen M (2018) The Length of Hydrophobic Chain in Amphiphilic Polypeptides Regulates the Efficiency of Gene Delivery. Polymers. https://doi.org/10.3390/polym10040379 


\section{Figures}

Fig. 1 (a) A photograph of TBA-TNS colloidal solution irradiated by laser light, (b) particle size distribution curves and (c) XRD patterns of TMA-TNS and TBA-TNS, and (d) a schematic illustration of $\mathrm{R}_{4} \mathrm{~N}-\mathrm{TNS}$ in a solution and after drying

Fig. 2 (a) Raman spectra of colloidal solutions of TMA-TNS and TBA-TNS and (b) calibration curves to determine concentration of $\mathrm{R}_{4} \mathrm{~N}^{+}$fabricated using characteristic peak areas of $\mathrm{TMA}^{+}\left(721-772 \mathrm{~cm}^{-1}\right)$ and $\mathrm{TBA}^{+}\left(850-964 \mathrm{~cm}^{-1}\right)$ marked as arrows in (a)

Fig. 3 Changes in turbidity of E. cloacae suspensions including (a) TMA-Cl, TBA-Cl, and (b) other $\mathrm{R}_{4} \mathrm{~N}^{+}$ with several alkyl chain lengths $\left(\mathrm{C}_{n} \mathrm{H}_{2 n+1} \mathrm{~N}^{+} \mathrm{Cl}^{-}, \mathrm{n}=2,3\right.$ and $\left.6,0.05 \mathrm{M}\right)$ during incubation at $37^{\circ} \mathrm{C}$ and $(\mathrm{c})$ a photograph of samples examined in (b) after incubation for $77 \mathrm{~h}$

Fig. 4 (a) Formed colonies on a LB agar plate and (b) the CFUs of free $\mathrm{R}_{4} \mathrm{~N}^{+}$(TMA-Cl, TBA-Cl) and TNS composites (TMA-TNS, TBA-TNS), and (c) the CFUs of comparison materials $\left(\mathrm{TiO}_{2}\right.$ and $\left.\mathrm{TiO}_{2} / \mathrm{TBA}-\mathrm{Cl}\right)($ $p \leq 0.001, * * * * p \leq 0.0001$ in relation to the control)

Fig. 5 Predicted mechanisms of antibacterial behavior using TBA-TNS and $\mathrm{TiO}_{2} / \mathrm{TBA}-\mathrm{Cl}$ 
Fig. 1
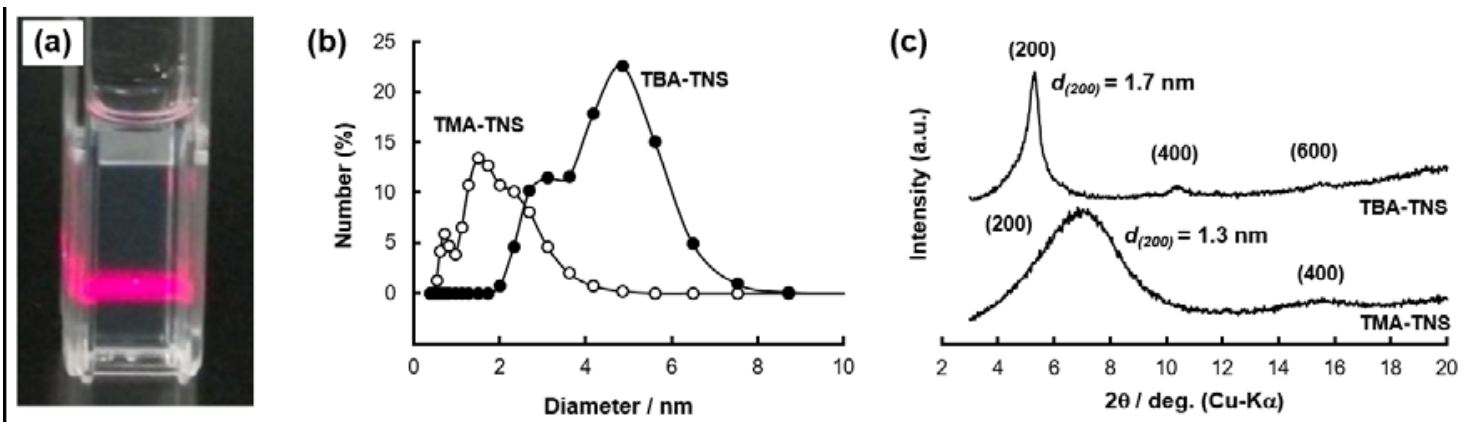

(d)

In solution (during DLS analysis)

After coated (during XRD analysis)

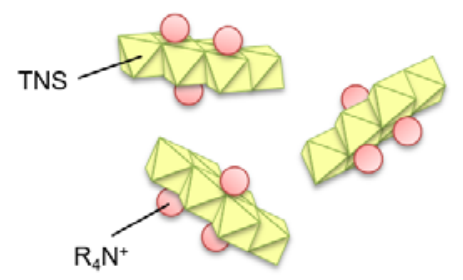

$\mp d$-spacing of (200)

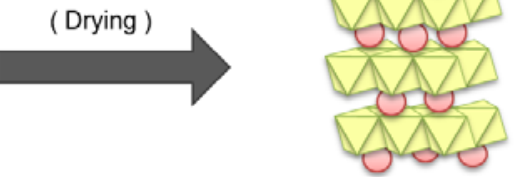

$\pm$ 
Fig. 2

(a)

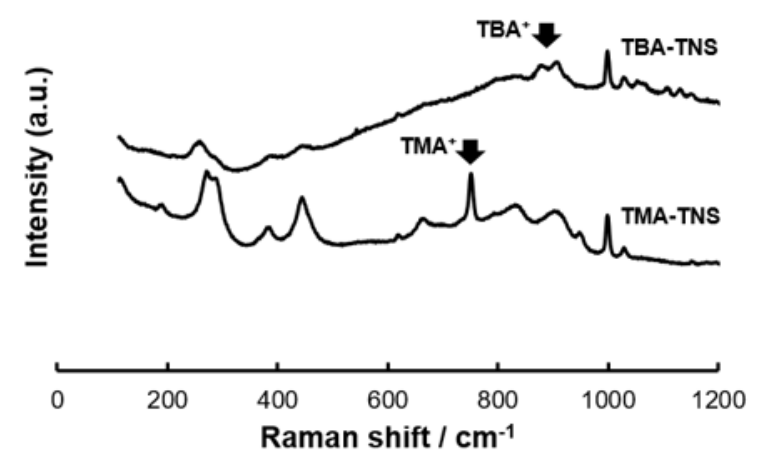

(b)

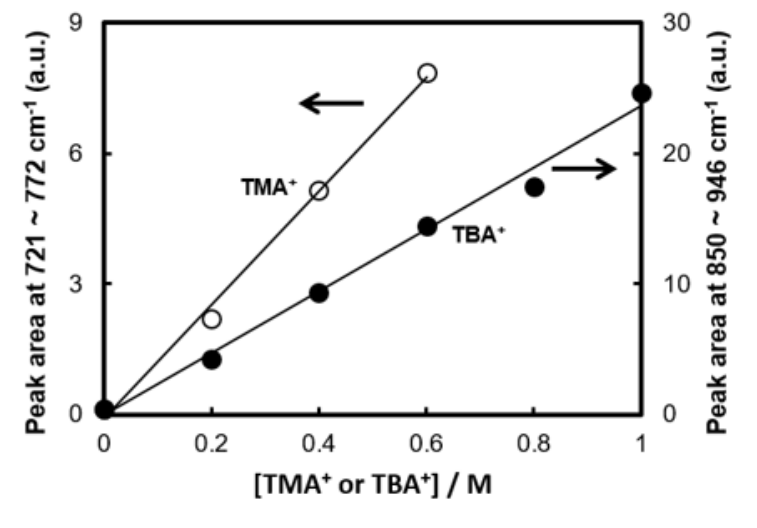


Fig. 3
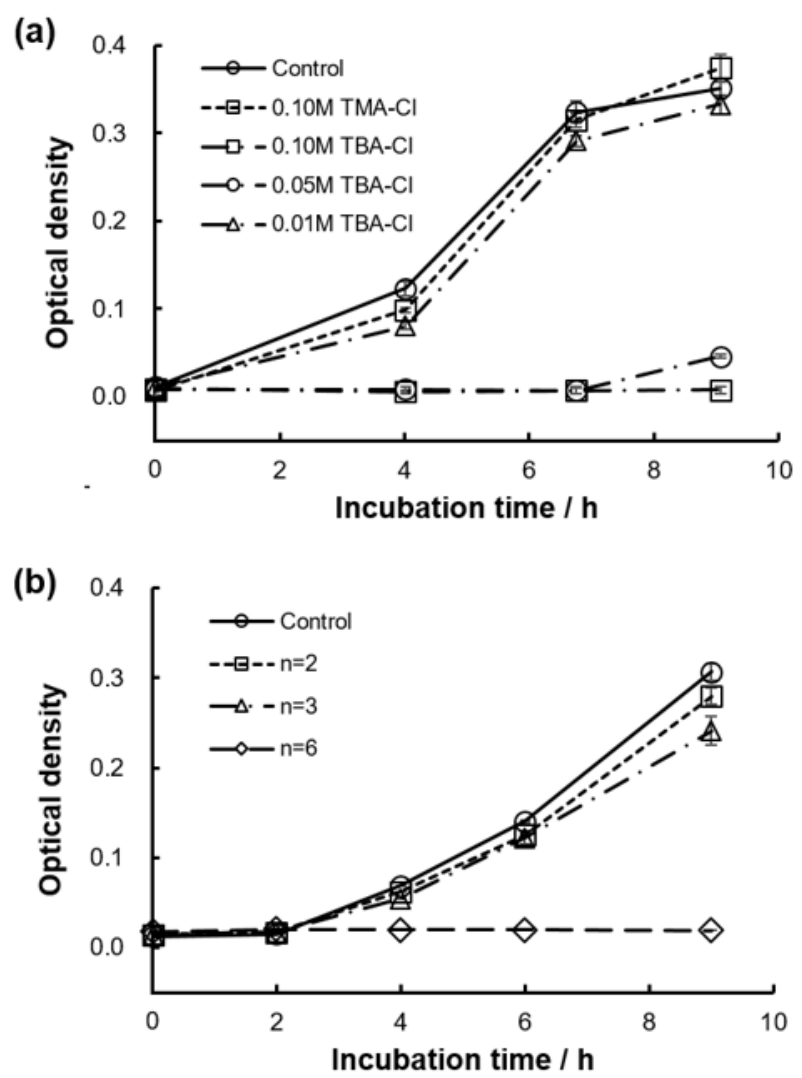

(c)

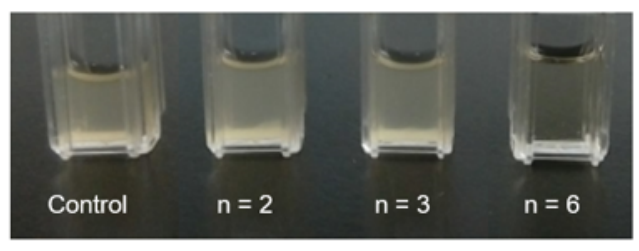


Fig. 4

(a)

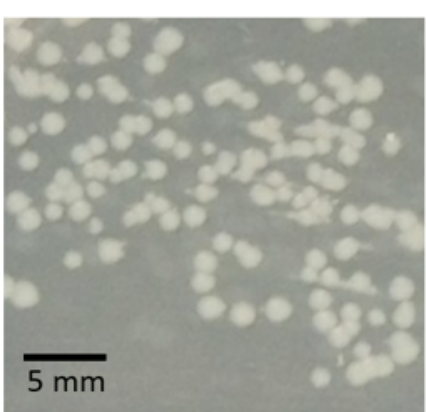

(b)

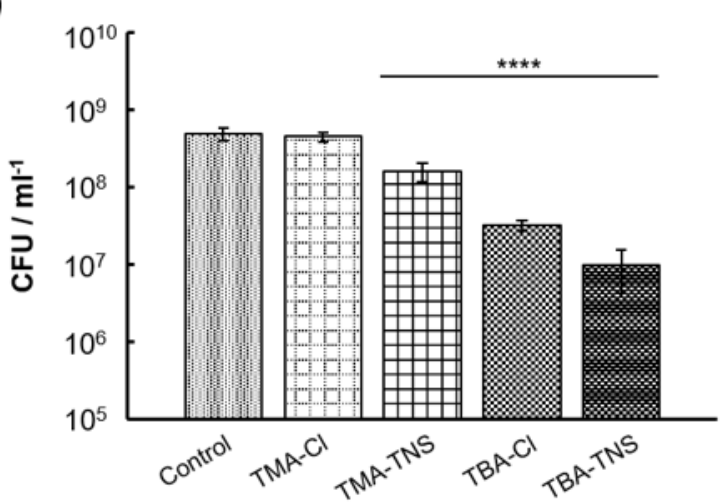

(c)

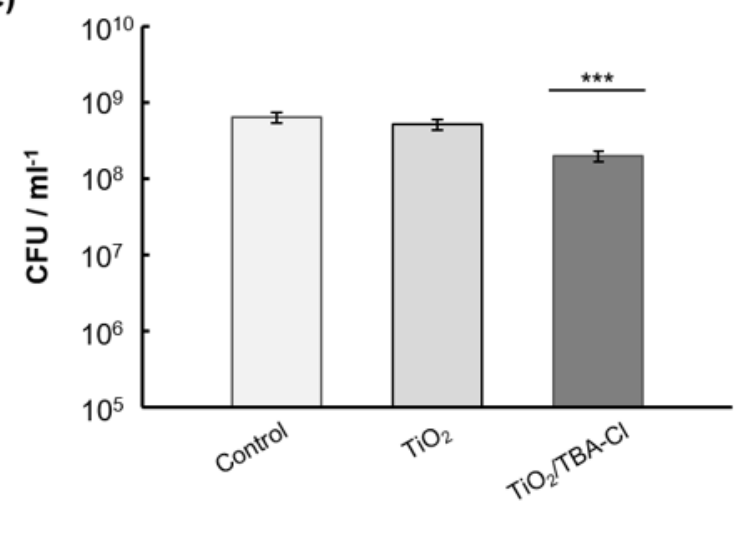


Fig. 5

TBA-TNS

(a)

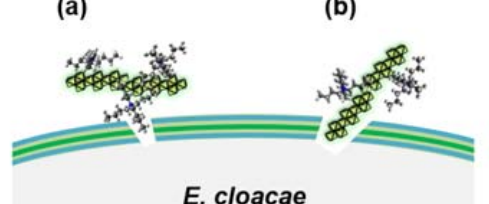

$\mathrm{TiO}_{2} / \mathrm{TBA}-\mathrm{Cl}$

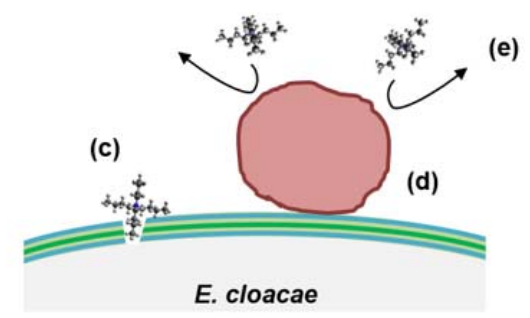

(e)

TNS

$\underset{4}{4}$ TBA $^{+}$

$\mathrm{TiO}_{2}$ 